\title{
SV2B is essential for the integrity of the glomerular filtration barrier
}

\author{
Yoshiyasu Fukusumi, Ayako Wakamatsu, Natsumi Takashima, Eriko Hasegawa, Naoko Miyauchi, \\ Masayuki Tomita and Hiroshi Kawachi
}

The glomerular visceral epithelial cell (podocyte) is characterized as a specialized structure of the interdigitating foot processes, covering the outer side of the glomerular basement membrane (GBM). The neighboring foot processes are connected by a slit diaphragm, which is a key structure regulating the barrier function of the glomerular capillary wall to prevent proteinuria. We have previously reported that synaptic vesicle protein 2 B (SV2B) is expressed in the podocyte and that the expression is clearly decreased in nephrotic models. However, the precise function of SV2B in the podocyte is unclear. To investigate the role of SV2B in maintaining the podocyte function and to better understand the function of the neuron-like vesicle expressing SV2B in the podocyte, we analyzed them with SV2B knockout (KO) mice. An increase in the amount of proteinuria, effacement of the foot process of the podocyte, and alterations of the GBM were detected in SV2B KO mice. It was also found that the expression of CD2AP, nephrin, and NEPH1, the functional molecules of the slit diaphragm, and laminin, a critical component of the GBM, is clearly altered in SV2B KO mice. Synaptotagmin and neurexin, which have a role in the synaptic vesicle docking in neurons, are downregulated in the kidney cortex of SV2B KO mice. We have previously reported that neurexin interacts with CD2AP, and the present study shows that SV2B interacts with CD2AP. These findings suggest that the SV2B-neurexin complex is involved in the formation and maintenance of the slit diaphragm. In addition, SV2B is densely expressed close to the cell surface in the presumptive podocyte in the early stage of glomerulogenesis. These results suggest that SV2B has an essential role in the formation and maintenance of the glomerular capillary wall.

Laboratory Investigation (2015) 95, 534-545; doi:10.1038/labinvest.2015.39; published online 2 March 2015

Proteinuria is not only an important symptom of kidney diseases but also an accelerating factor progressing to endstage renal failure. Recent studies showed that proteinuria is also a risk factor for cardiac and cranial vascular events. The glomerulus, a tiny, ball-shaped structure composed of capillaries, is a filtration unit of the kidney, and the glomerular capillary wall functions as a barrier, preventing the leakage of plasma protein into urine. The glomerular capillary wall consists of three layers: an endothelial cell, a glomerular basement membrane (GBM), and a glomerular visceral epithelial cell (podocyte). The main body of the GBM is a meshwork of type IV collagen, and several molecules such as laminin are reported to be critical molecules in the GBM. ${ }^{1}$ A podocyte is characterized as a specialized structure of the interdigitating foot processes, covering the outer side of the GBM. The neighboring foot processes are derived from different cell bodies and are connected by a continuous membrane-like structure called a slit diaphragm..$^{2-4}$ It is now widely accepted that the slit diaphragm is a key structure regulating the barrier function of the glomerular capillary wall and that the dysfunction of the slit diaphragm is involved in the development of proteinuria in several types of kidney disease. Although the dysregulation of the barrier function of the glomerular capillary wall causes serious clinical outcomes, there is little knowledge on the mechanism of the formation and the maintenance of its structure.

We have previously reported that synaptic vesicle protein 2 B (SV2B), which is an isoform of SV2 and was originally identified as a membrane protein on a synaptic vesicle, is expressed in the podocyte and that the expression is clearly decreased in nephrotic models. ${ }^{5} \mathrm{We}$ also showed that CD2AP, which is one of the critical molecules of the slit diaphragm and is detected at the processes of the normal cultured podocyte, is not detected at the process area of the cultured

Department of Cell Biology, Institute of Nephrology, Niigata University Graduate School of Medical and Dental Sciences, Niigata, Japan

Correspondence: Professor H Kawachi, MD, PhD, Department of Cell Biology, Institute of Nephrology, Niigata University Graduate School of Medical and Dental Sciences, 1-757 Asahimachi-dori, Chuo-ku, Niigata 951-8510, Japan.

E-mail: kawachi@med.niigata-u.ac.jp

Received 8 September 2014; revised 12 December 2014; accepted 17 December 2014 
podocyte in which SV2B has been knocked down. ${ }^{5}$ These observations suggest that SV2B is a functional molecule of the podocyte and may have a role in the trafficking of CD2AP forward to the tip of processes. However, the precise function of SV2B in the podocyte has not been elucidated.

The expression of SV2B is detected in several organs and tissues, and histological studies show that SV2B is localized on a neuron-like vesicle, which shares many protein components with the synaptic vesicles of neurons. Rastaldi $e t a l^{6}$ reported that Rab3a, a synaptic vesicle molecule, is expressed on the vesicle surface in the podocyte. We have previously shown that SV2B is colocalized with Rab3a. ${ }^{5}$ These observations suggest that SV2B in the podocyte is expressed on the surface of the neuron-like vesicle. However, the function of the neuron-like vesicle in the podocyte is not well understood.

In the present study, to further investigate the role of SV2B in maintaining the podocyte function and to better understand the function of the neuron-like vesicle expressing SV2B in the podocyte, we analyzed the phenotypes of SV2B knockout (KO) mice, the interaction of SV2B with other podocyte functional molecules, and the developmental expression of SV2B. An increase in the amount of proteinuria, effacement of the foot process of the podocyte, and alterations of the GBM were detected in SV2B KO mice. It was also found that the expression of CD2AP, nephrin, and NEPH1, the functional molecules of the slit diaphragm, and laminin, a critical component of GBM, was clearly altered in SV2B KO mice. In addition, synaptotagmin and neurexin, which have a role in the synaptic vesicle docking in neurons, were downregulated in the kidney cortex of SV2B KO mice. We also show here that SV2B interacts with CD2AP and nephrin, and that SV2B is densely expressed close to the cell surface in the presumptive podocyte in the early developmental stage of glomeruli. It is conceivable that these results indicate that SV2B has an essential role in the formation and maintenance of the glomerular capillary wall.

\section{MATERIALS AND METHODS \\ Animals}

SV2B-mutant mice (129P2/OlaHsd background $)^{7}$ were generated from frozen sperm from Jackson Laboratory (Stock No. 006383). SV2B KO mice were obtained by mating SV2B heterozygous mice. A PCR for genotyping was performed using three primers used at Jackson Laboratory. The common primer (5'-GGCACTCAGCCACTAACTCTCAGTACA-3'), a wild-type (WT) reverse primer (5'-TCATCCAGATGATGTC AAGTCTAAGC- $3^{\prime}$ ), and a mutant reverse primer (5'-GAG CGCGCGCGGCGGAGTTGTTGAC-3'), which bound a site located within the neomycin-resistant gene, were used. Mouse tails were lysed in an STE buffer (10 mM Tris- $\mathrm{HCl}(\mathrm{pH} 8.0)$, $100 \mathrm{mM}$ methylenediaminetetraacetic acid (EDTA), 0.5\% sodium dodecyl sulfate) containing $500 \mu \mathrm{g} / \mathrm{ml}$ proteinase $\mathrm{K}$ (Sigma-Aldrich, St Louis, MO, USA) at $55^{\circ} \mathrm{C}$ overnight with shaking. A genomic PCR was performed according to Jackson Laboratory's protocol.

A total of seven KO mice (two 19-week-old mice, three 30week-old mice, one 37-week-old mouse, and one 44 week-old mouse) and age-matched five WT mice (two 19-week-old mice, one 32-week-old mouse, one 37-week-old mouse, and one 44-week-old mouse) were used. Twenty-four-hour urine samples were collected in metabolic cages just before killing, and their protein concentrations were measured by the Bradford method as described previously. ${ }^{8}$ For immunofluorescence (IF) analyses, five KO mice (19-, 19-, 30-, 37-, and 44-week-old) and the age-matched five WT mice were used. For light microscopy analysis, five KO mice (19-, 19-, 30-, 37-, and 44-week-old) and the age-matched five WT mice were used. For electron microscopy (EM) analysis, three KO mice (19-, 37-, 44-week-old) and the age-matched three WT mice were used. For real-time PCR analysis, five $\mathrm{KO}$ mice (19-, 19-, 30-, 37-, and 44-week-old) and the age-matched five WT mice were used.

For dual-labeling IF, Duolink in situ assays, and an immunoprecipitation assay, three female Wistar rats purchased from Charles River Japan (Atsugi, Japan) were used. Kidneys were removed from these rats, and the pieces of each rat were used for dual-labeling IF and Duolink in situ assays. The remaining kidneys of these rats were pooled, and glomeruli were isolated from these kidneys by a sieving method, solubilized with an SDS-PAGE sample buffer, and used for an immunoprecipitation assay.

For the developmental study, a pair of Wistar rats was purchased from Charles River Japan, and mated, and the kidneys were removed from three embryos.

All animal experiments conformed to the National Institutes of Health Guide for the care and Use of Laboratory Animals. Procedures for the present study were approved by the Animal Committee according to the guidelines for animal experimentation of Niigata University.

\section{IF Study}

The IF studies were performed basically according to the method previously reported. ${ }^{8,9}$ A rabbit anti-nephrin antibody, ${ }^{8}$ mouse anti-nephrin antibody, ${ }^{10}$ rabbit antipodocin antibody, ${ }^{9}$ rabbit anti-CD2AP antibody, ${ }^{11}$ rabbit anti-NEPH1 antibody, ${ }^{12}$ and rabbit anti-neurexin antibody ${ }^{13}$ were prepared in the authors' laboratory. The specificity of the rabbit anti-nephrin antibody, rabbit anti-podocin antibody, rabbit anti-NEPH1 antibody, and rabbit anti-neurexin antibody was confirmed by the absorption test with specific antigens. ${ }^{8,9,12,13}$ The specificity of the mouse monoclonal anti-nephrin antibody was proved by the crosslinking method. ${ }^{8}$ The specificity of the rabbit anti-CD2AP antibody was confirmed by the absorption test with a GST fusion protein of full-length CD2AP, which was used for immunization. The findings of the absorption test are shown in Supplementary Figure S2. Rabbit anti-podocalyxin was prepared in rabbits immunized with podocalyxin-rich fraction prepared 
with WGA-sepharose 4B according to the method described by Kerjaschki et al. ${ }^{14}$ Rabbit anti-SV2A and anti-SV2B antibodies were purchased from Synaptic Systems (Goettingen, Germany). Goat anti-CD2AP antibody and goat antiNHERF2 antibody were purchased from Santa Cruz Biotechnology (Dallas, TX, USA). Rabbit anti-integrin antibody was purchased from Millipore (Billerica, MA, USA). Rabbit anti-collagen IV antibody was purchased from Cosmo Bio (Tokyo, Japan). Rabbit anti-laminin antibody was purchased from Sigma-Aldrich. Rabbit anti-ZO-1 antibody was purchased from Invitrogen (Camarillo, CA, USA). Rabbit polyclonal anti-fibronectin antibody was generated against human fibronectin as immunogen. TRITC-conjugated antimouse Igs and FITC-conjugated anti-rabbit Igs were purchased from DakoCytomation (Glostrup, Denmark). TRITC-conjugated anti-goat IgG was purchased from Protos Immunoresearch (Burlingame, CA, USA). IF staining for CD2AP, nephrin, NEPH1, podocin, ZO-1, integrin, collagen IV, fibronectin, laminin, podocalyxin, and NHERF2 was semiquantified basically according to the method described by Macconi et al $^{15}$ (score 0 , completely absent; 1, signal covering $0-25 \%$ of the glomerular tuft area; 2, 25-50\%; 3, 50$75 \%$; and $4,75-100 \%)$. IF staining for neurexin was evaluated as the extension of the linear pattern. The ratio (\%) of glomeruli showing a continuous linear pattern along the capillary wall in whole glomerulus relative to the total number of glomeruli was observed. The stainings were evaluated in 30 glomeruli of each mouse in a blinded manner. The data are expressed as mean \pm s.d. of five mice of each genotype.

\section{Primary Cultured Cells of Glomeruli}

The kidney cortex from WT or SV2B KO mice was minced, and glomeruli were isolated under the stereomicroscope. The isolated glomeruli were cultured in an RPMI 1640 medium (Nissui Pharmaceutical, Tokyo, Japan) supplemented with $10 \%$ fetal bovine serum (Life Technologies, Grand Island, NY, USA) at $37^{\circ} \mathrm{C}$ in an atmosphere of 5\% CO2 and $100 \%$ humidity. After four days, cells were fixed, incubated with a rabbit anti-CD2AP antibody or rabbit anti-neurexin antibody, and stained with FITC-conjugated anti-rabbit Igs.

\section{Western Blot Analysis}

Western blot analysis with the kidney cortex lysates of WT or SV2B KO mice was performed basically according to the method described previously. ${ }^{12}$ The strips of the membrane were exposed to a rabbit anti-phospho-PI3K p85 antibody or rabbit anti-PI3K p85 antibody (Cell Signaling Technology, Danvers, MA, USA). The stripes were washed and incubated with alkaline phosphatase-conjugated anti-rabbit immunoglobulins (Zymed Laboratory Inc. San Francisco, CA, USA).

\section{Evaluation of the Width of Foot Process}

The width of foot process was evaluated by the method of Bechtel et al. ${ }^{16}$ Randomly selected five glomeruli of $n=3$ mice each were evaluated. EM images were analyzed using ImageJ software. From each picture, the mean width of the foot processes (FPWs) was calculated according to the following formula: $\mathrm{FPW}=\pi / 4 \times(\Sigma \mathrm{GBM}$ length $/ \Sigma$ foot process $)$. A foot process was defined as any connected epithelial segment butting on the basement membrane between two neighboring filtration slits.

\section{Real-Time RT-PCR}

The total RNA was extracted from kidney cortex with TRIzol (Life Technologies). Two micrograms of RNA was used to synthesize cDNA with SuperScript II (Life Technologies BRL) according to the manufacturer's protocol. Synthesized cDNA was used as a template. cDNA, specific primers, and SYBR Green (Takara, Otsu, Japan) were mixed with a Takara Ex Taq R-PCR Version For Real Time PCR kit (Takara). PCR reactions were run on a Smart Cycler System (Takara). Ct values of the gene targets were normalized to glyceraldehyde3-phosphate dehydrogenase (GAPDH). Fold change in expression of target genes compared with the WT mice was calculated using the 2- $\Delta \Delta \mathrm{CT}$ method with GAPDH. The sequences of the primers were shown in Supplementary Table S2.

\section{Immunoprecipitation Assay}

Immunoprecipitation assays were performed basically according to the method described previously. ${ }^{13} \mathrm{~A}$ rat glomerular lysate was incubated with an anti-SV2B antibody at $4{ }^{\circ} \mathrm{C}$ overnight and precipitated with Dynabeads Protein G (Invitrogen, Carlsbad, CA, USA) in the tube. The tube was placed on the magnet, and the supernatant was removed. The Dynabeads-antibody-antigen complex was washed five times with PBS containing $0.1 \%$ Triton X-100, and then the antigen was eluted with an SDS-PAGE sample buffer. The eluates were separated by SDS-PAGE followed by immunoblotting with an anti-CD2AP antibody or anti-nephrin antibody. Alkaline phosphatase-conjugated rabbit anti-goat immunoglobulins (Zymed Laboratory) were used to detect CD2AP or nephrin.

\section{Duolink In Situ Assay}

A Duolink in situ assay, which can detect a protein-protein interaction, ${ }^{17}$ was performed with Duolink in situ PLA probes purchased from Olink Bioscience, Uppsala, Sweden. The principal of this assay is as follows: two primary antibodies raised in different species were used. Species-specific secondary antibodies, called PLA probes, with a unique short DNA strand bind to the primary antibodies. When the PLA probes are in close proximity $(<40 \mathrm{~nm})$, the DNA strands can interact. After amplification, the DNA circle is detected with a fluorescence microscope. The analysis was performed according to the manufacturer's protocol. In brief, rat kidney sections were incubated with rabbit anti-SV2B and mouse anti-nephrin, and the interaction was analyzed with Duolink in situ PLA probe anti-rabbit PLUS and Duolink in situ PLA probe anti-mouse MINUS. Similarly, the rat kidney sections 
a
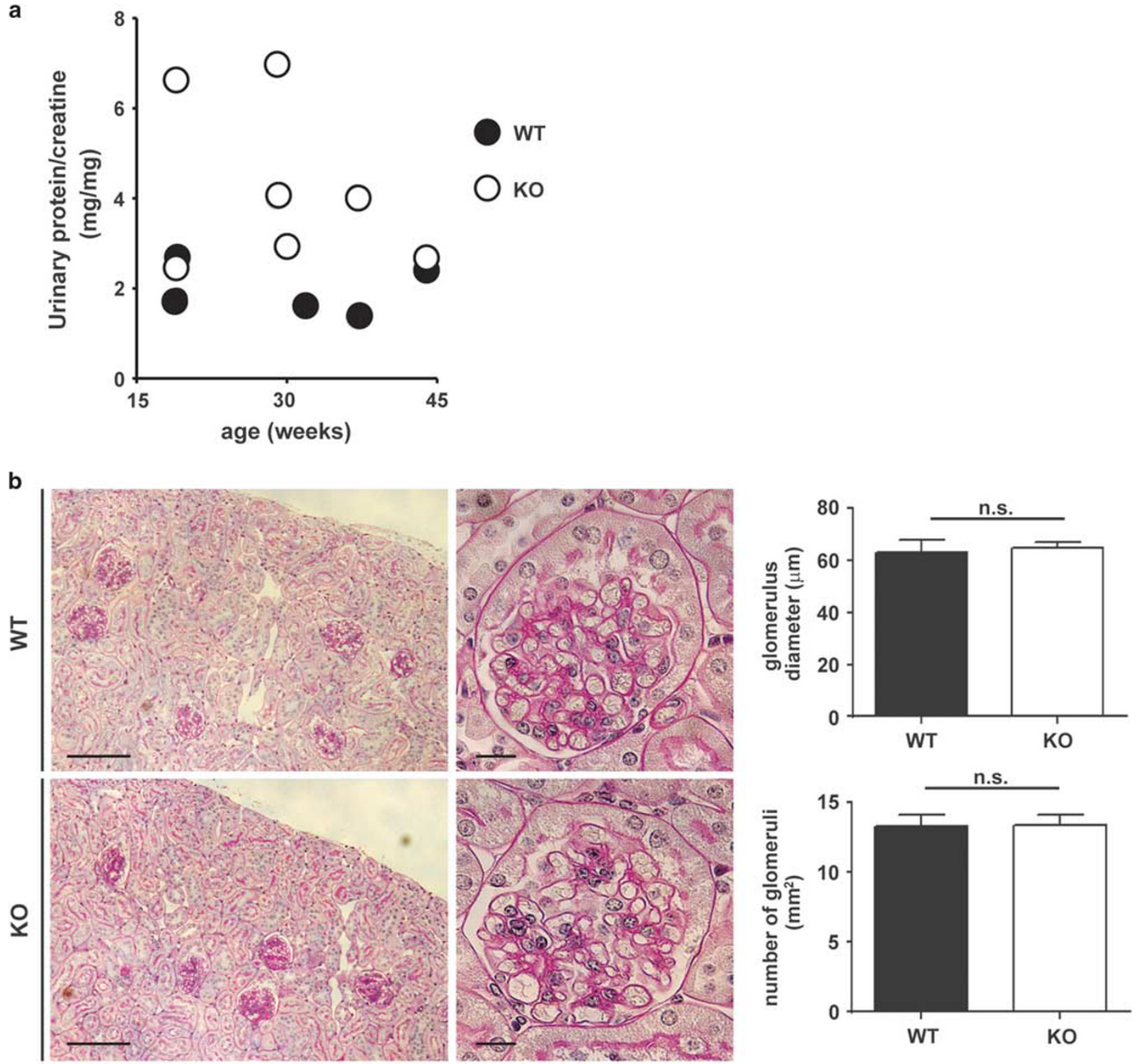

Figure 1 SV2B deficiency causes proteinuria, podocyte foot process effacement, and glomerular basement thickness. (a) Urinary protein excretion was increased in KO mice. $n=5$ WT mice, $n=7$ KO mice. (b) Kidney sections of WT and KO mice did not display significant differences in glomerular diameter or number of glomeruli. Scale bars, $100 \mu \mathrm{m}$ in left panels; $20 \mu \mathrm{m}$ in right panels. $n=5$ per group, n.s., not significant, two-tailed $t$-test. (c) Electron microscopy of KO mice showed foot process effacement (arrows) and GBM thickness (asterisks). FPW, foot process width. Scale bars, $5 \mu \mathrm{m}$ in left panels; $1 \mu \mathrm{m}$ in right panels. $n=3$ per group, ${ }^{*} P<0.05,{ }^{*} P<0.01$, two-tailed $t$-test.

were incubated with rabbit anti-SV2B and goat anti-CD2AP, and the interaction was analyzed with Duolink in situ PLA probe anti-rabbit PLUS and Duolink in situ PLA probe antigoat MINUS.

\section{Statistical Analyses}

Statistical significance was evaluated using the unpaired $t$-test or the Mann-Whitney $U$-test. Values were expressed as the mean \pm s.d. Differences at $P<0.05$ were considered significant. Data were analyzed using Graphpad Prism 5.0 software (Graphpad Software, San Diego, CA, USA).

\section{Results}

\section{SV2B KO Mice Show Proteinuria and Morphological} Alterations

To elucidate the function of SV2B in vivo, we generated SV2B KO mice from frozen sperm from Jackson Laboratory (Supplementary Figure S1a-d). SV2B KO mice were born without body-weight loss or any external abnormality 

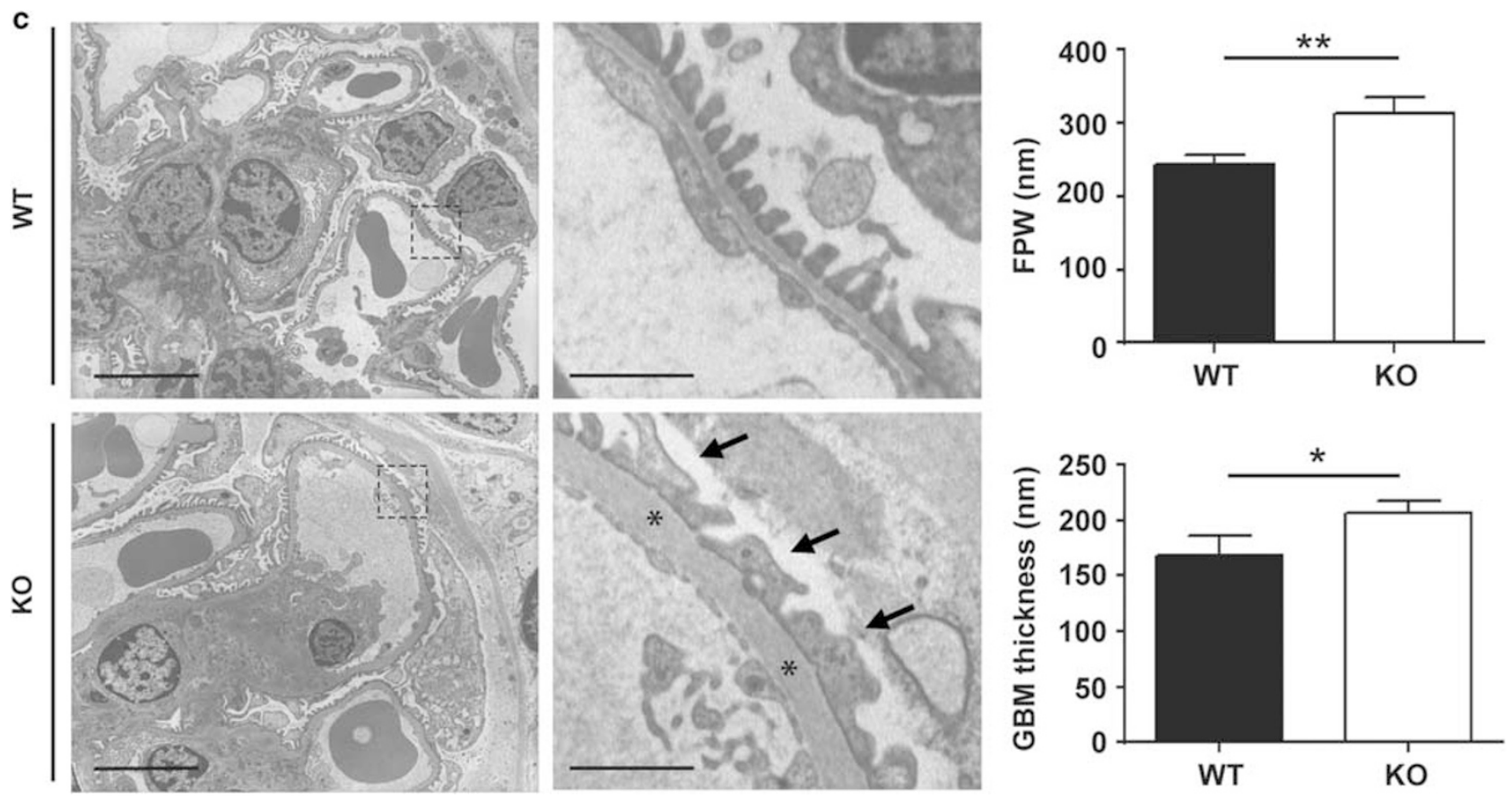

Figure 1 (Continued).

(Supplementary Table S1). An increase in the amount of proteinuria was detected in SV2B KO mice (Figure 1a). The number and size of glomeruli in SV2B KO mice were similar to those in WT mice (Figure 1b). An EM analysis revealed effacement of the foot process with significantly increased foot process widths and an irregular and thickened GBM in the KO mice (Figure 1c).

\section{Defect of SV2B Leads to the Altered Expression of Slit Diaphragm and GBM Components}

We analyzed the expression of the podocyte functional molecules and the GBM components in SV2B KO mice. $\mathrm{CD} 2 \mathrm{AP}$ staining in glomeruli and primary cultured cells of glomeruli was clearly altered in the SV2B KO mice (Figure 2a and Supplementary Figure 4). Alterations in the staining patterns of nephrin and NEPH1 were also detected (Figure 2b and c). The expression of other slit diaphragm molecules, podocin and ZO-1, was not altered (Figure $2 \mathrm{~d}$ and e). Alterations in the expression of neither synaptopodin (Figure 2f), an actin-associated molecule, nor podocalyxin (Figure 3a) and NHERF2 (Figure 3b), apical surface molecules, were observed in SV2B KO mice. The expression of integrin, a basal surface molecule connecting the foot process to the GBM by binding to laminin, a component of the GBM, was not altered in SV2B KO mice (Figure 3c). Next, we analyzed GBM components in SV2B KO mice. The staining of laminin was clearly reduced in SV2B KO mice (Figure 3f). Alterations in the expression of other major GBM components, collagen type IV and fibronectin, were not detected (Figure 3d and e).

\section{Defects in SV2B Do Not Affect the PI3K Pathway}

To elucidate whether SV2B is involved in PI3K pathway, we analyzed the expression of phosphorylated PI3K p85 in the kidney cortex lysates of SV2B KO mice. No differences of the expression levels of phosphorylated PI3K p85 were detected. The findings were shown in Supplementary Figure S3. The results suggested the deficiency of SV2B does not affect PI3K pathway.

\section{SV2B Is Colocalized with the Slit Diaphragm Markers}

A dual-labeling IF study with normal adult rat kidney sections showed that SV2B was diffusely expressed in glomeruli, and the dense dot staining of SV2B was colocalized with CD2AP (Figure 4a) and nephrin (Figure 4b). Duolink in situ analysis ${ }^{17}$ revealed that SV2B lay in close proximity to CD2AP and nephrin (Figure 4c).

\section{SV2B Interacts with Slit Diaphragm Molecules}

The specific bands of nephrin and CD2AP were detected in the normal rat glomerular lysates precipitated with the antiSV2B antibody (Figure 4d).

\section{Defect of SV2B Leads to the Downregulation of Synaptic Vesicle-Associated Molecules}

To elucidate the relationship of SV2B with other synaptic vesicle-associated molecules, we analyzed their expression by real-time PCR in the kidney cortex of SV2B KO mice. The expression of SV2A and SV2C, other isoforms of SV2, was not altered in the SV2B KO mice. No alterations in the mRNA expression of other synaptic vesicle surface proteins, Rab3a 
a
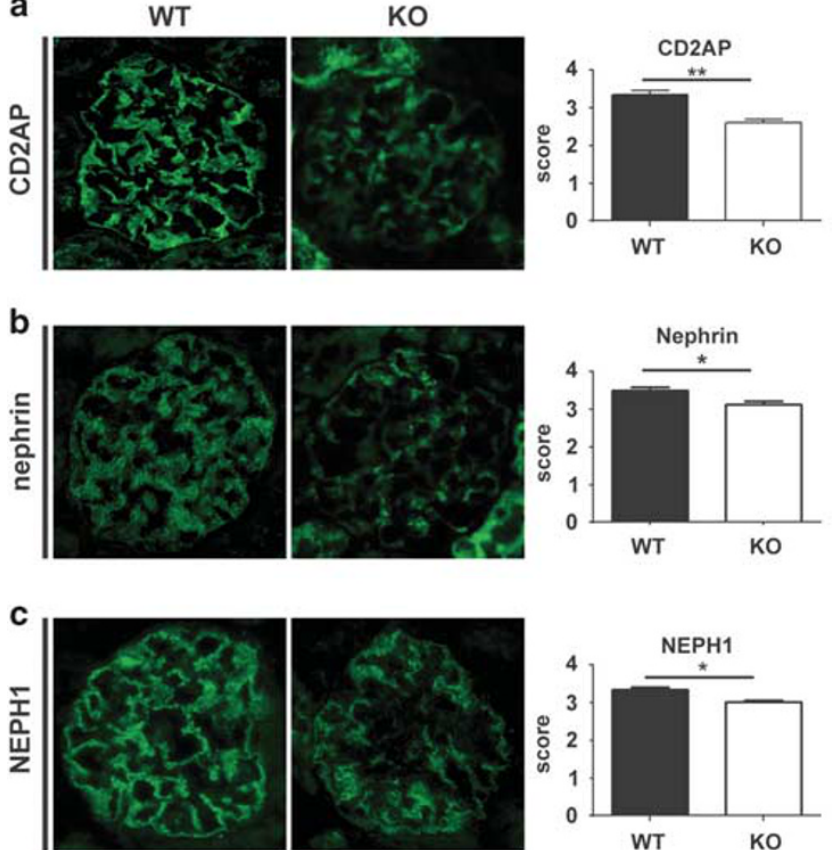
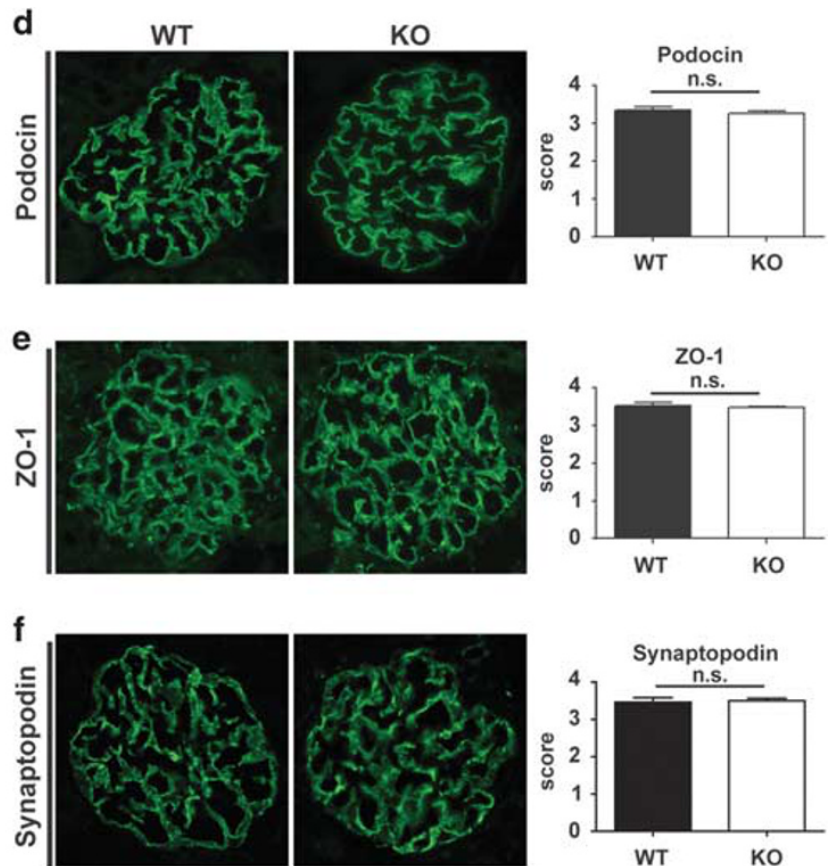

Figure 2 Representative immunofluorescence images of kidney sections and semiquantitative analysis of the stainings of the slit diaphragm-associated proteins in SV2B KO mice. Staining of CD2AP (a), nephrin (b), and NEPH1 (c) in KO mice was significantly decreased, but podocin (d), ZO-1 (e) and synaptopodin (f) stainings were not altered. $n=5$ per group, n.s., not significant, ${ }^{* *} P<0.01,{ }^{*} P<0.05$, Mann-Whitney test.
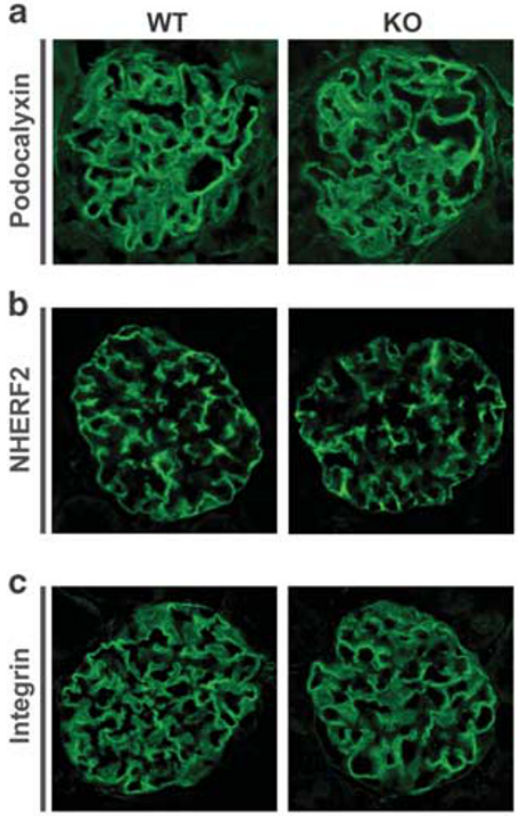
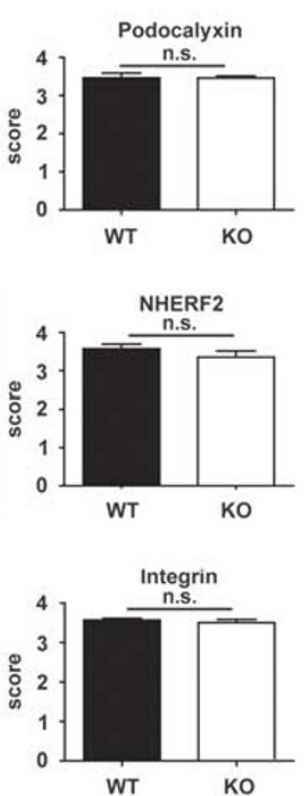
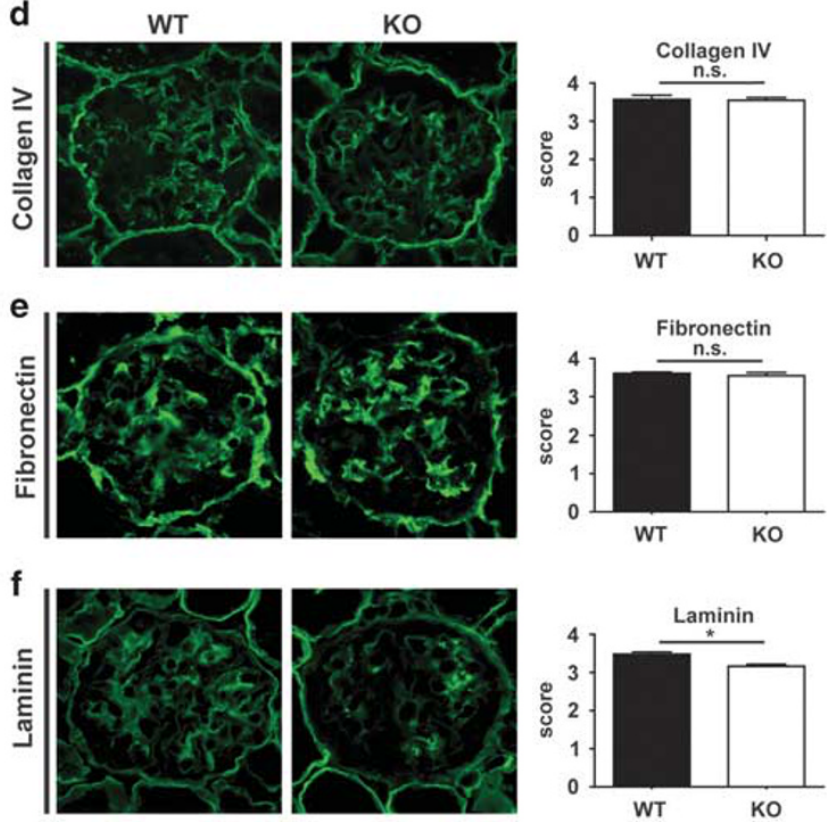

Figure 3 Representative immunofluorescence images of kidney sections and semiquantitative analysis of the stainings of the apical, basal surface molecules and glomerular basement membrane molecules in SV2B KO mice. Alterations of stainings of podocalyxin (a), nor NHERF2 (b), the apical surface molecules in podocytes, were not observed in KO mice. The staining of integrin, a basal surface molecule in podocyte, was not altered in KO mice (c). Although collagen IV (d) and fibronectin (e) stainings were not altered, staining of laminin (f) was significantly decreased in KO mice. $n=5$ per group, n.s., not significant, ${ }^{*} P<0.05$, Mann-Whitney test.

and vGlut1, were detected in the SV2B KO mice. On the other hand, the expression of synaptotagmin, a $\mathrm{Ca}^{2+}$ sensor in the membrane of the presynaptic axon terminal, and neurexin, a presynaptic adhesion molecule in the neuron, were clearly reduced in the kidney cortex of SV2B KO mice (Figure 5a). Because we previously reported that neurexin localizes at the slit diaphragm of the glomerular podocytes in kidneys, ${ }^{13}$ we analyzed the immunostaining of neurexin in SV2B KO mice. 
a

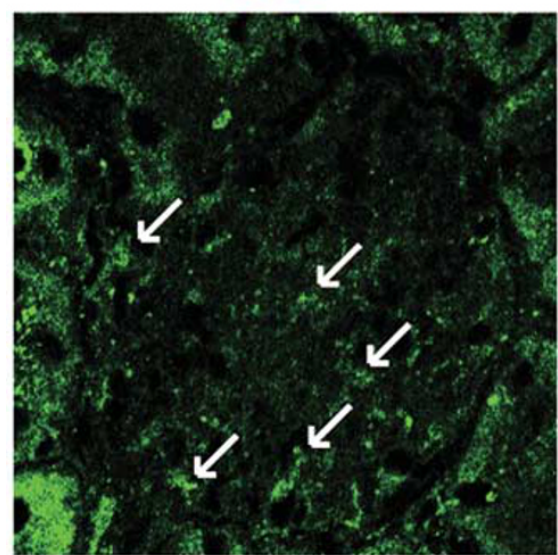

b

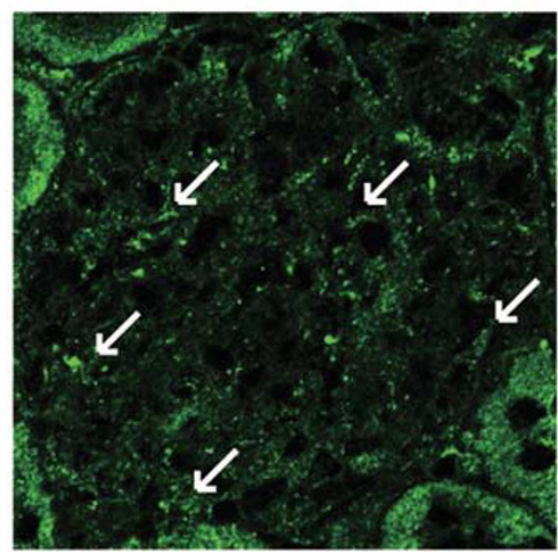

c

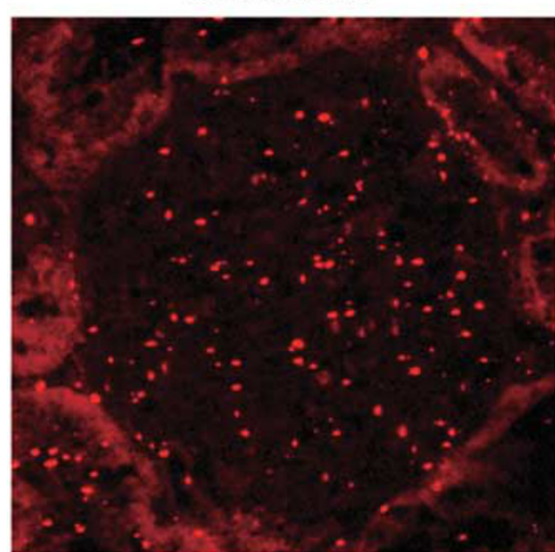

CD2AP

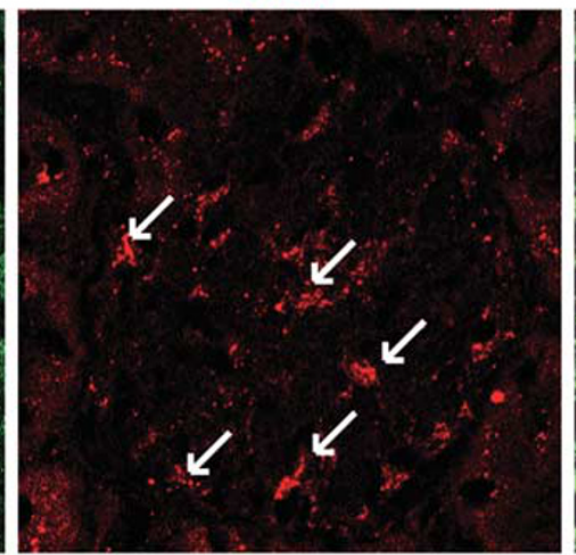

Nephrin

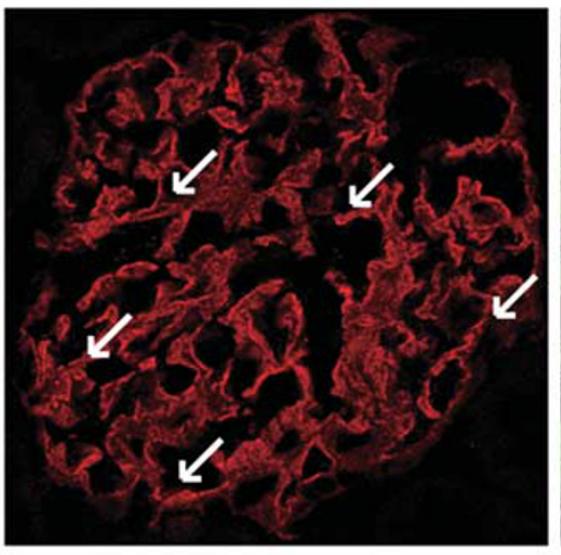

SV2B/Nephrin

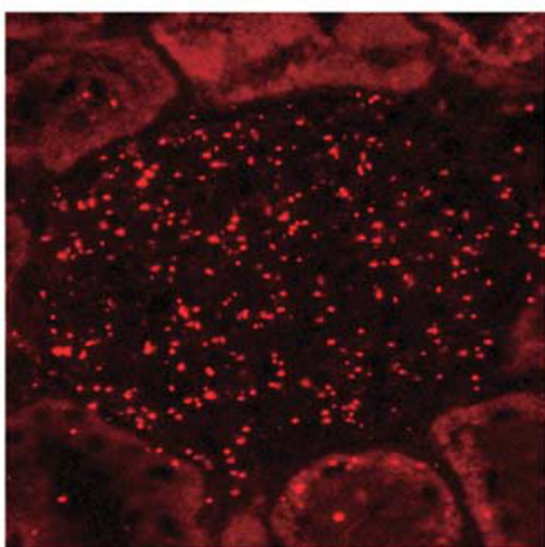

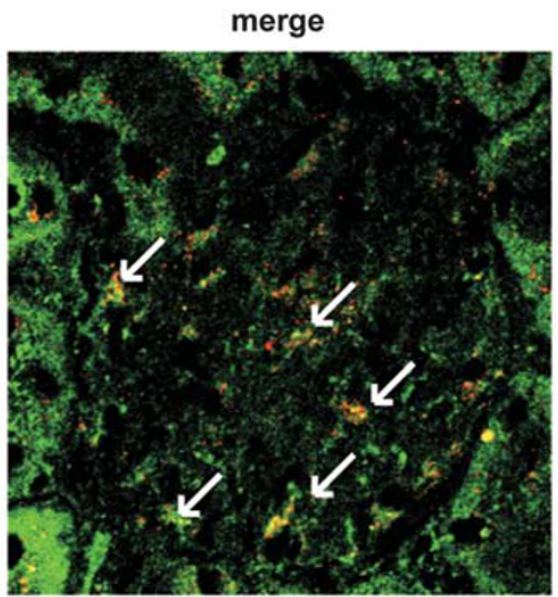

merge

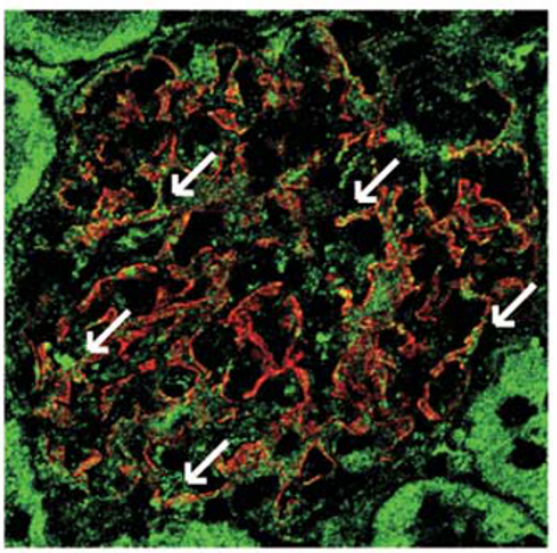

SV2B only

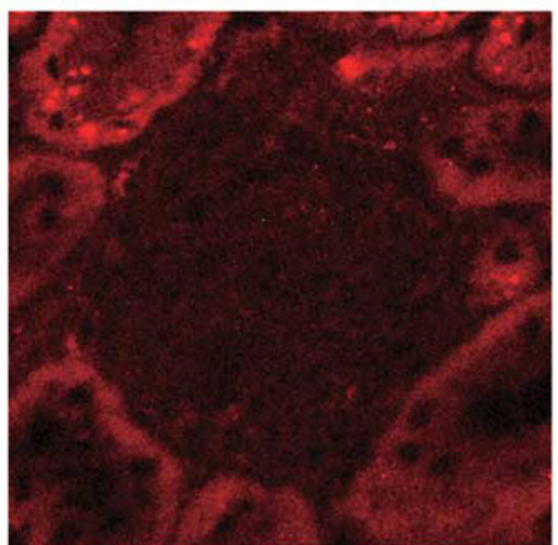

Figure 4 SV2B is colocalized and interacts with the slit diaphragm molecules in rat glomeruli. (a) Confocal immunofluorescence microscopy showed that SV2B was colocalized with CD2AP (arrows). (b) SV2B was closely localized with nephrin (arrows). (c) Duolink in situ analysis showed that SV2B lay in close proximity to CD2AP and nephrin as red dot signals in glomeruli. As a negative control, only the anti-SV2B antibody was incubated. (d) In the immunoprecipitation assay, the bands of CD2P and nephrin were detected in the rat glomerular lysate precipitated with an anti-SV2B antibody.

Neurexin in glomeruli was observed as a linear pattern along the capillary wall in WT mice, whereas the staining intensity of neurexin was clearly reduced and its pattern changed to a discontinuous dot pattern in $\mathrm{KO}$ mice (Figure 5b). Although the staining of neurexin in primary cultured cells of glomeruli from WT mice was detected at the processes of cells, which of neurexin in primary cultured cells of glomeruli from $\mathrm{KO}$ mice was clearly lower than that of WT mice (Supplementary 
Figure S4). Neurexin colocalized with CD2AP in rat glomeruli (Figure 5c). Dual-labeling IF showed that neurexin was colocalized with CD2AP. The Duolink method showed that neurexin localized close to CD2AP (Figure 5d).

d
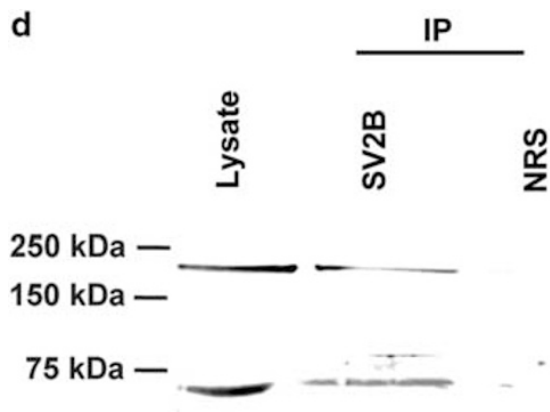

Nephrin

CD2AP

Figure 4 (Continued)

SV2B Is Clearly Expressed along the Whole-Cell Surface
of the Podocyte at the Early Stage of Glomerulogenesis To understand the role of SV2B in podocyte differentiation, we analyzed the expression of SV2B in immature podocytes in developing glomeruli with rat kidney sections on embryonic day 20.5. The staining of SV2B was restricted in glomeruli (Figure 6a), whereas the staining of SV2A was broadly detected in kidney sections (Figure 6b). The SV2B staining was already detected along the whole-cell surface of the presumptive podocyte of the early S-shaped body stage glomeruli when nephrin expression was not yet detected.

\section{Discussion}

It has been pointed out that neurons and podocytes have common characteristics. Both are terminally differentiated cells and have similar shapes characterized by processes. ${ }^{18,19}$ Both of them also display cell type-specific intercellular contact: synapses in neurons and the slit diaphragm in podocytes. Furthermore, functionally important molecules in the podocyte, such as nephrin, podocin, and neurexin, have

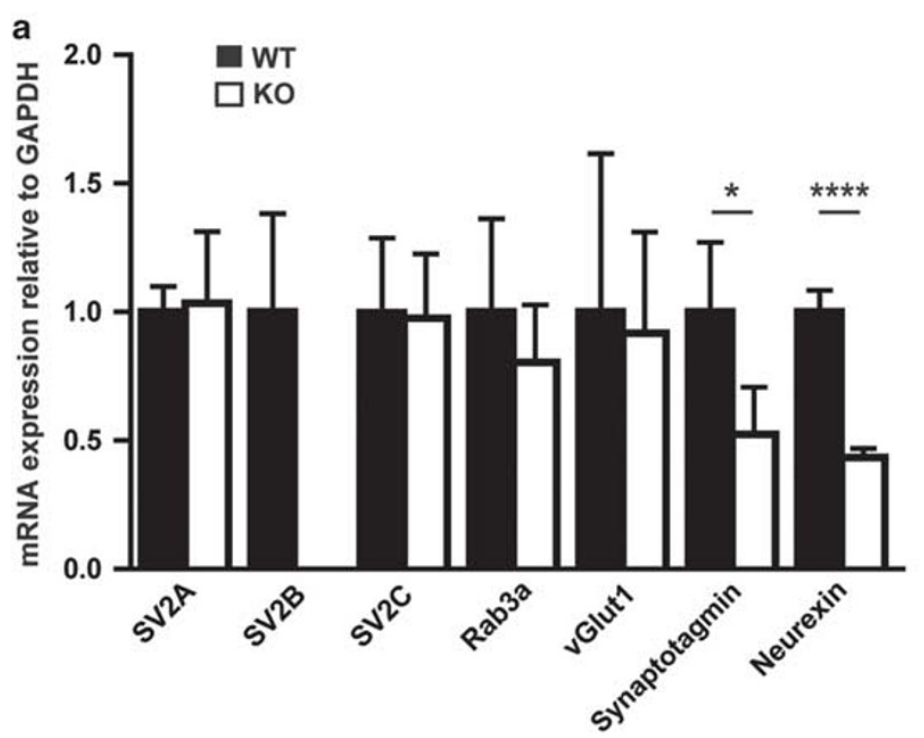

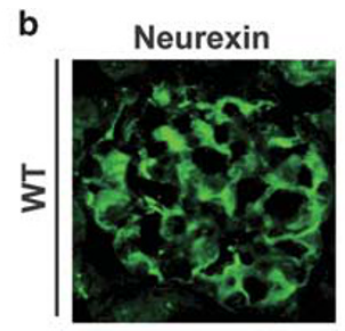
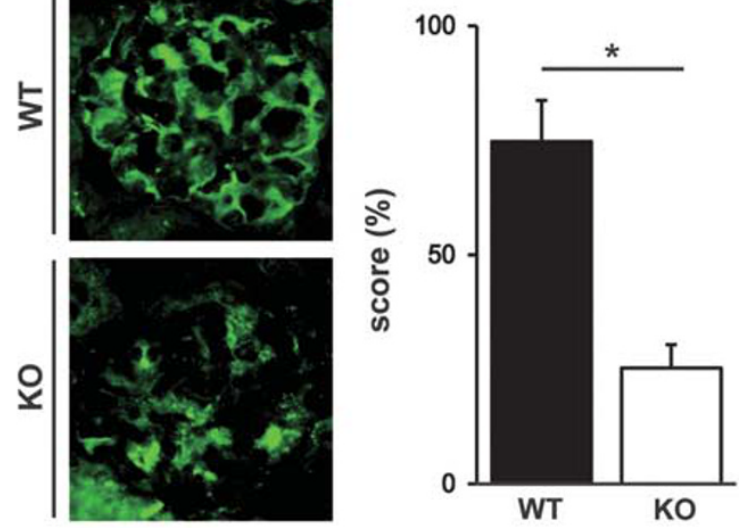
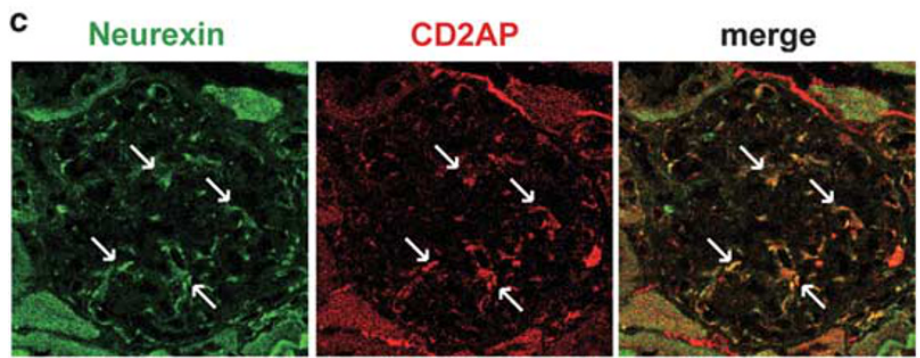

d
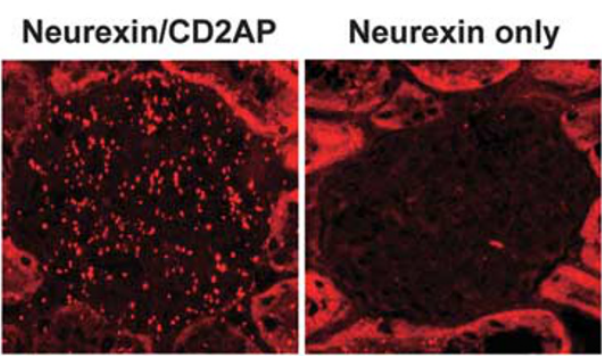

Figure 5 Analyses of the expression of synaptic vesicle-associated molecules in SV2B KO mice. (a) Real-time PCR from the mouse kidney cortex showed that the mRNA expression of synaptotagmin and neurexin were decreased in KO mice. No alterations in the mRNA expression of other synaptic vesicle surface proteins, SV2A, SV2C, Rab3a and vGlut1, were detected in the KO mice. $n=5$ per group, ${ }^{*} P<0.05$, ${ }^{* * * *} P<0.0001$, two-tailed $t$-test. (b) Staining of neurexin was decreased in KO mice. $n=5$ per group, ${ }^{*} P<0.05$, Mann-Whitney test. (c) Confocal immunofluorescence microscopy revealed that neurexin was colocalized with CD2AP (arrows). (d) Duolink in situ analysis showed that neurexin localized close to CD2AP. Neurexin only is a negative control (right panel). 


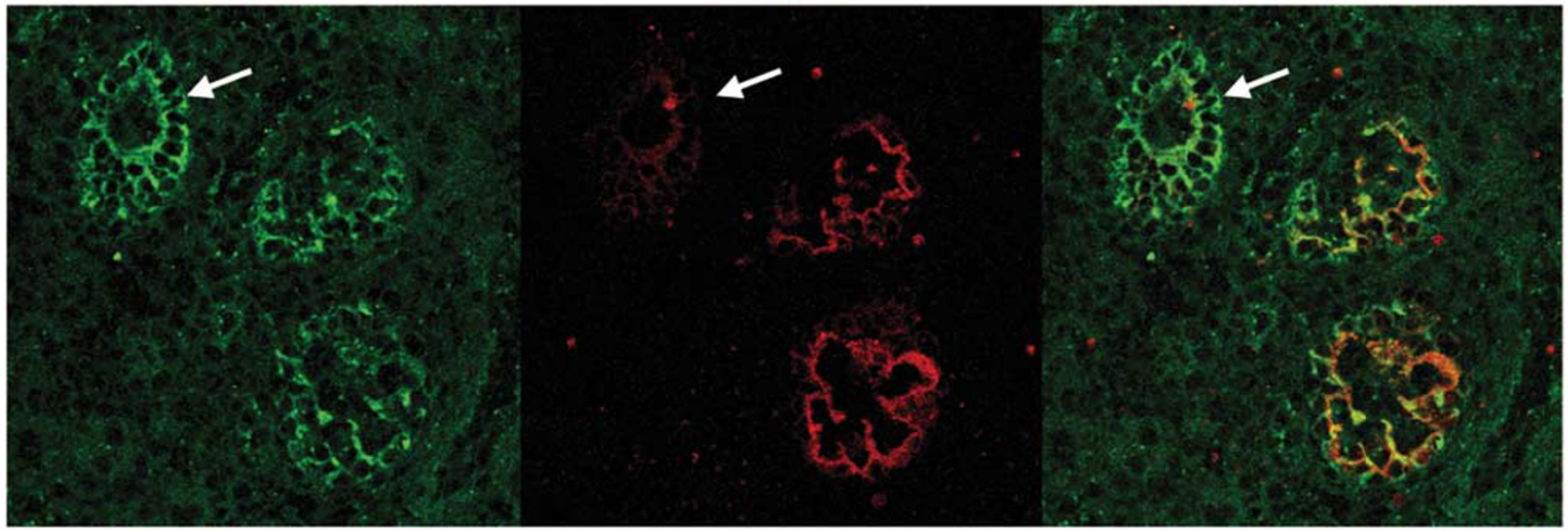

b

SV2A

Nephrin

merge

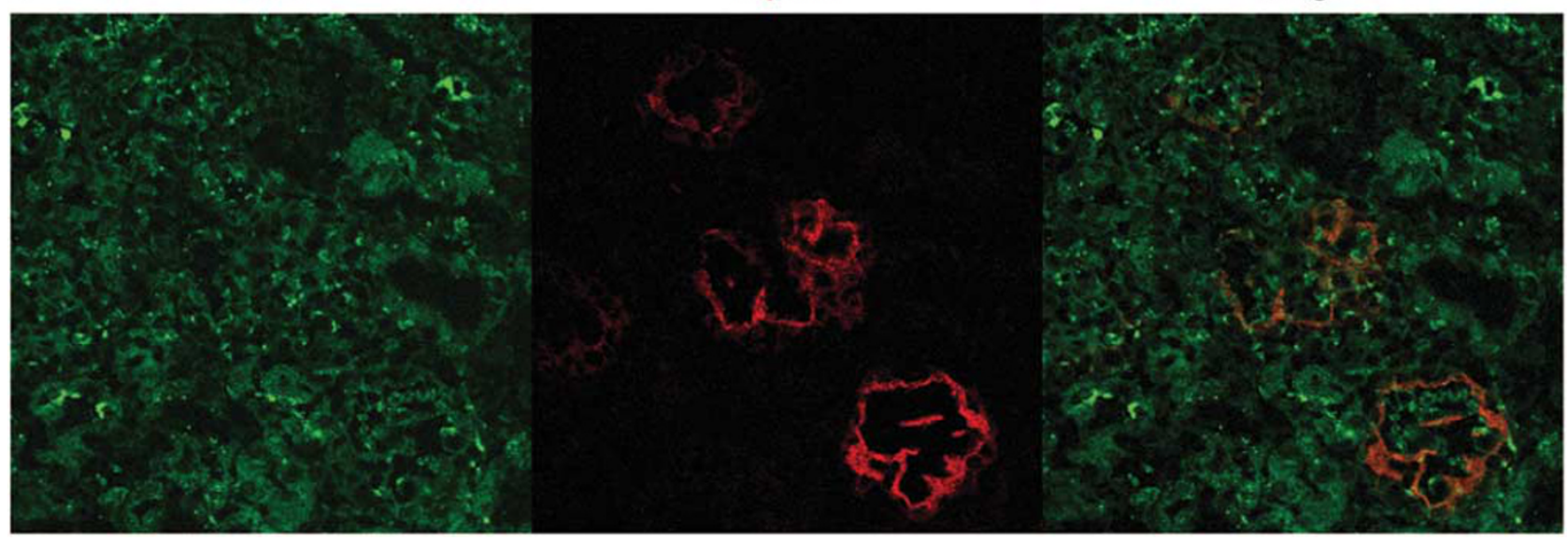

Figure 6 Dual-labeling immunofluorescence findings of SV2B and SV2A with nephrin in the developing glomeruli of embryonic day 20.5 rat kidney sections. (a) The staining of SV2B was restricted in glomeruli. The SV2B staining was already detected along the whole-cell surface of the presumptive podocyte of the early S-shaped body stage glomeruli when nephrin expression was not yet detected (arrows). (b) The staining of SV2A was broadly detected in kidney sections.

been found to be specifically shared with the neuron. ${ }^{8,9,20}$ It has been reported that Rab3a, a synaptic vesicle molecule, is expressed on the surface of the vesicle in the podocyte, and it is proposed that the podocyte contains neuron-like functional vesicles. ${ }^{6}$ Because we observed that SV2B is colocalized with Rab3a, ${ }^{5}$ SV2B in the podocyte is considered to be expressed on the surface of the neuron-like vesicle. In the present study, we showed that SV2B KO mice display an increased amount of proteinuria; disarrangement of the slit diaphragm molecules, CD2AP, nephrin, and NEPH1 (Figure 2), and of the GBM component laminin (Figure 3); and alterations of the foot processes and irregular thickened structure of the GBM (Figure 1). We also observed that the staining of CD2AP was negative at the processes of primary cultured cells of glomeruli from SV2B KO mice, although clear CD2AP staining was detected at those of cultured cells from WT mice (Supplementary Figure S4). Although, we analyzed these phenotypes of the mice at the age of 19-44-week-old in this study, we did not detect the change in the phenotypes vary with age. These phenotypes of SV2B KO mice in glomeruli suggest that SV2B in the podocyte has a role to maintain the structure of the glomerular capillary wall.

The alteration in the distribution of CD2AP was dominant rather than other slit diaphragm molecules, nephrin and NEPH1, of which expression was altered in SV2B KO mice (Figure 2). The altered distribution of CD2AP in the cultured podocyte treated with siRNA for SV2B was observed in the previous study. ${ }^{5}$ In addition, in this study we showed that SV2B had an interaction with CD2AP (Figure 5). On the basis of these observations, it is conceivable that SV2B regulates the expression of CD2AP. Li et al ${ }^{21}$ reported that CD2AP KO mice displayed the alteration of nephrin expression. CD2AP is a cytoplasmic component of the slit diaphragm, which connects the cytoskeletal actin and nephrin, a transmembrane protein constituting the filter structure of the extracellular site of the slit diaphragm. It is also reported that nephrin has an 
interaction with NEPH1, another extracellular component of the slit diaphragm. ${ }^{22}$ Thus, it is conceivable that the CD2AP alteration resulting from the defect of SV2B leads to the altered localization of nephrin and NEPH1, although the precise mechanism by which the defect of SV2B brings about the disarrangement of these slit diaphragm molecules is unclear. It should be mentioned that the alterations of podocin and ZO-1, other critical components of the slit diaphragm, are not detected in SV2B KO mice. These are cytoplasmic proteins localized at the insertion point of the slit diaphragm. ZO-1 was originally identified as a component of tight junction and is also detected at the slit diaphragm. ${ }^{23,24}$ ZO-1 is reported to connect actin and NEPH1. ${ }^{25}$ The findings in SV2B KO mice suggested that the regulation of the localization of podocin and ZO-1 is independent of that of CD2AP-nephrin-NEPH1. No alterations in the expression of other podocyte functional molecules, synaptopodin, an actinassociated molecule, podocalyxin and NHERF2, apical surface molecules, or integrin, a basal surface molecule connecting to the GBM, were observed in SV2B KO mice (Figure 3). These observations indicated that SV2B specifically regulated the localizations of CD2AP-nephrin-NEPH1.

In this study, we also found the reduced staining of laminin and the GBM abnormality in SV2B KO mice, although the alterations in the expression of other GBM components were not detected (Figure 3). It is understood that major components of the GBM, including laminin, were synthesized by the podocyte and that laminin is involved in the link between the basal side of the podocyte and the GBM. Morgans et al reported that the loss of SV2B results in reduced neurotransmission and altered synaptic vesicle protein expression in the retina. They concluded that SV2B contributes to the modulation of synaptic vesicle exocytosis and has a significant role in regulating synaptic protein content. ${ }^{26}$ SV2B in the podocyte might be involved in the exocytosis for laminin.

It has been reported that nephrin and CD2AP interact with the p85 subunit of $\mathrm{PI} 3 \mathrm{~K}^{27}$ and that the PI3K pathway is involved in actin reorganization and cell survival in podocytes. ${ }^{28,29}$ To elucidate whether SV2B is involved in the PI3K pathway, we analyzed the expression of phosphorylated PI3K p85 in the kidney cortex lysates of SV2B KO mice. The alterations of phosphorylated PI3K p85 or total PI3K were not observed in SV2B KO mice (Supplementary Figure S3), suggesting that the deficiency of SV2B does not affect the PI3K pathway.

Next, to better understand the nature of the vesicle mediated by SV2B, we elucidated the relationship of SV2B with other synaptic vesicle-associated molecules by analyzing their expression in the kidney cortex of SV2B KO mice. We analyzed the expression of SV2A, SV2C, Rab3a, vGlut1, and synaptotagmin, which are supposed to be expressed on the vesicle surface, and neurexin, which is reported to be expressed on the cell surface and to participate in the docking of the vesicle to the cell membrane (Figure 5a). The expression of all synaptic vesicle-associated molecules examined in this study was detected in the kidney cortex, a glomeruli-rich area, of WT mice. The alterations in the expression of SV2A and SV2C were not detected in SV2B KO mice, suggesting that SV2B has no functional association with other isoforms of SV2 in the vesicle of the podocyte. In this study, the altered expression of neither Rab3a nor vGlut1 was detected in SV2B $\mathrm{KO}$ mice. It is reported that Rab3a mediated the exocytosis for glutamate in the podocyte. ${ }^{30} \mathrm{SV} 2 \mathrm{~B}$ might not be involved in the Rab3a-mediated vesicle function in the podocyte.

We found that the expression of synaptotagmin, which acts as a $\mathrm{Ca}^{2+}$ sensor on the synaptic vesicle in the neuron, was clearly decreased in the kidney cortex of SV2B KO mice (Figure 5a). It was reported that SV2B KO mice showed a reduced expression of synaptotagmin in the retina, of which synapses express only the SV2B isoform. ${ }^{26,31}$ The study in retinae also showed that SV2B has a direct interaction with synaptotagmin and regulates the stability of synaptotagmin. ${ }^{31}$ Studies with the hippocampal neuron showed that SV2 has a critical role in the trafficking of synaptotagmin to the synaptic vesicle. $^{32}$ Our study and previous reports suggest that SV2B has a functional association with synaptotagmin both in the synaptic vesicle of the neuron and in the neuron-like vesicle of the podocyte. In the podocyte, transient receptor potential channel 6 (TRPC6), a non-selective cation channel, is reported to be localized at the slit diaphragm and to have a critical role in regulation of the $\mathrm{Ca}^{2+}$ level. ${ }^{33,34}$ It is reported that strict regulation of the $\mathrm{Ca}^{2+}$ level is important for the stable expression of slit diaphragm molecules. ${ }^{35}$ SV2B may participate in regulation of the $\mathrm{Ca}^{2+}$ level in cooperation with synaptotagmin.

In this study, we have also found that the expression of neurexin in the podocyte is clearly reduced in SV2B KO mice (Figure $5 \mathrm{a}$ and $\mathrm{b}$ ). The decreased staining of neurexin was also observed in the primary culture of the podocyte from SV2B KO mice. Neurexin is a presynaptic adhesion molecule binding to the postsynaptic adhesion molecule neuroligin. Some studies on the neuron suggested that synaptotagmin is involved in the docking of the synaptic vesicles via neurexin. ${ }^{36,37}$ Recently, we have shown that neurexin localized at the slit diaphragm and that the expression of neurexin was clearly reduced in disease models showing proteinuria. ${ }^{13}$ On the basis of these observations, we proposed that neurexin is one of the functional components of the slit diaphragm. In the previous report, we also showed that CD2AP was co-precipitated with an anti-neurexin antibody and that the staining of CD2AP almost completely overlapped with neurexin by dual-labeling immunohistochemical analysis. ${ }^{13}$ In the present study, we showed that CD2AP was detected in the precipitate with the anti-SV2B antibody and that major portions of CD2AP staining were co-stained with SV2B (Figure 4). On the basis of these observations, it is conceivable that neurexin interacts with the SV2B-CD2AP complex in the podocyte. 
To elucidate the role of SV2B in the maturation of the podocyte, the expression of SV2B in the developing glomeruli was analyzed. Although the staining of SV2A was broadly detected in kidney sections, the staining of SV2B was restricted in the podocytes of the developing glomeruli (Figure 6). The differential expression also supports that not SV2A but SV2B has a major role in the podocyte. It is reported that Rab3a expression is first detected in completely matured glomeruli. ${ }^{38}$ The staining of SV2B is already detected at the early S-shaped body stage glomeruli, when nephrin expression was not yet detected. The positive staining of SV2B was detected along the whole-cell surface of the presumptive podocyte. With maturation of the podocyte, the SV2B staining came to be concentrated on the basolateral surface near the slit diaphragm. The dense and broad expression of SV2B along the whole-cell surface of the presumptive podocyte suggested that SV2B is involved in the maturation of the podocyte, although the role of SV2B detected at the apical area of the maturing podocyte is unclear.

In this study, we propose a model in which SV2B has a major role in regulating the expression of synaptotagmin on neuron-like vesicles in the podocyte. The SV2B and synaptotagmin complex interacts with neurexin at the slit diaphragm, and this molecular complex has a role in the docking of the vesicle and in the regulation of the $\mathrm{Ca}^{2+}$ level at the terminus of the foot processes where the slit diaphragm is located. It is conceivable that the SV2B/synaptotagmin/ neurexin complex is involved in the formation and maintenance of the slit diaphragm by regulating the strict arrangement of CD2AP, nephrin, and NEPH1. SV2B is also involved in the exocytosis for laminin (Supplementary Figure S5). The present study implies that SV2B has an important role in the maintenance of the filtration barrier of the glomerular capillary wall and that the dysfunction of SV2B participates in the development of proteinuria. Dysregulation of the synaptic vesicle function is understood to be involved in several neuronal diseases, and the drug targeting the synaptic vesicle is used for treatment of epilepsy. SV2B and other synaptic vesicle-associated molecules could be a novel therapeutic target for nephrotic syndrome.

Supplementary Information accompanies the paper on the Laboratory Investigation website (http://www.laboratoryinvestigation.org)

\section{ACKNOWLEDGMENTS}

This work was supported by Grant-Aids for Scientific Research (B: 23390224 to HK), (C: 23591186 to MT) and Grant-Aid for Young Sciences (B: 24790839 to YF) from the Ministry of Education, Culture, Sports, Science, and Technology of Japan. We thank Mutsumi Kayaba and Yukina Kitazawa for excellent technical assistance, and Masaaki Nameta for taking EM images.

\section{DISCLOSURE/CONFLICT OF INTEREST}

The authors declare no conflict of interest.

1. Miner JH. The glomerular basement membrane. Exp Cell Res 2012;318: 973-978.
2. Kawachi H, Matsui K, Orikasa $M$ et al. Quantitative studies of monoclonal antibody 5-1-6-induced proteinuric state in rats. Clin Exp Immunol 1992;87:215-219.

3. Kriz W, Shirato I, Nagata M et al. The podocyte's response to stress: the enigma of foot process effacement. Am J Physiol Renal Physiol 2013;304:F333-F347.

4. Pavenstadt H, Kriz W, Kretzler M. Cell biology of the glomerular podocyte. Physiol Rev 2003;83:253-307.

5. Miyauchi N, Saito A, Karasawa $T$ et al. Synaptic vesicle protein $2 B$ is expressed in podocyte, and its expression is altered in proteinuric glomeruli. J Am Soc Nephrol 2006;17:2748-2759.

6. Rastaldi MP, Armelloni S, Berra S et al. Glomerular podocytes possess the synaptic vesicle molecule Rab3A and its specific effector rabphilin3a. Am J Pathol 2003;163:889-899.

7. Janz R, Goda Y, Geppert M et al. SV2A and SV2B function as redundant Ca2+ regulators in neurotransmitter release. Neuron 1999;24:1003-1016.

8. Kawachi $\mathrm{H}$, Koike $\mathrm{H}$, Kurihara $\mathrm{H}$ et al. Cloning of rat nephrin: expression in developing glomeruli and in proteinuric states. Kidney Int 2000;57: 1949-1961.

9. Kawachi $\mathrm{H}$, Koike $\mathrm{H}$, Kurihara $\mathrm{H}$ et al. Cloning of rat homologue of podocin: expression in proteinuric states and in developing glomeruli. J Am Soc Nephrol 2003;14:46-56.

10. Orikasa M, Matsui $K$, Oite $T$ et al. Massive proteinuria induced in rats by a single intravenous injection of a monoclonal antibody. J Immunol 1988;141:807-814.

11. Nakatsue T, Koike H, Han GD et al. Nephrin and podocin dissociate at the onset of proteinuria in experimental membranous nephropathy. Kidney Int 2005;67:2239-2253.

12. Otaki Y, Miyauchi N, Higa M et al. Dissociation of NEPH1 from nephrin is involved in development of a rat model of focal segmental glomerulosclerosis. Am J Physiol Renal Physiol 2008;295:F1376-F1387.

13. Saito A, Miyauchi N, Hashimoto $T$ et al. Neurexin-1, a presynaptic adhesion molecule, localizes at the slit diaphragm of the glomerular podocytes in kidneys. Am J Physiol Regul Integr Comp Physiol 2011; 300:R340-R348.

14. Kerjaschki D, Sharkey DJ, Farquhar MG. Identification and characterization of podocalyxin-the major sialoprotein of the renal glomerular epithelial cell. J Cell Biol 1984;98:1591-1596.

15. Macconi D, Bonomelli M, Benigni A et al. Pathophysiologic implications of reduced podocyte number in a rat model of progressive glomerular injury. Am J Pathol 2006;168:42-54.

16. Bechtel W, Helmstadter M, Balica J et al. Vps34 deficiency reveals the importance of endocytosis for podocyte homeostasis. J Am Soc Nephrol 2013;24:727-743.

17. Soderberg $\mathrm{O}$, Gullberg $\mathrm{M}$, Jarvius $\mathrm{M}$ et al. Direct observation of individual endogenous protein complexes in situ by proximity ligation. Nat Methods 2006;3:995-1000.

18. Kobayashi N. Mechanism of the process formation; podocytes vs neurons. Microsc Res Tech 2002;57:217-223.

19. Kobayashi N, Gao SY, Chen J et al. Process formation of the renal glomerular podocyte: is there common molecular machinery for processes of podocytes and neurons? Anat Sci Int 2004;79:1-10.

20. Plaisier E, Mougenot B, Verpont MC et al. Glomerular permeability is altered by loss of P0, a myelin protein expressed in glomerular epithelial cells. J Am Soc Nephrol 2005;16:3350-3356.

21. Li C, Ruotsalainen V, Tryggvason $\mathrm{K}$ et al. CD2AP is expressed with nephrin in developing podocytes and is found widely in mature kidney and elsewhere. Am J Physiol Renal Physiol 2000;279:F785-F792.

22. Donoviel DB, Freed DD, Vogel $\mathrm{H}$ et al. Proteinuria and perinatal lethality in mice lacking NEPH1, a novel protein with homology to NEPHRIN. Mol Cell Biol 2001;21:4829-4836.

23. Kawachi $H$, Kurihara $H$, Topham PS et al. Slit diaphragm-reactive nephritogenic MAb 5-1-6 alters expression of ZO-1 in rat podocytes. Am J Physiol 1997;273(6 Pt 2):F984-F993.

24. Schnabel E, Anderson JM, Farquhar MG. The tight junction protein ZO-1 is concentrated along slit diaphragms of the glomerular epithelium. J Cell Biol 1990;111:1255-1263.

25. Huber TB, Schmidts M, Gerke $P$ et al. The carboxyl terminus of Neph family members binds to the PDZ domain protein zonula occludens-1. J Biol Chem 2003;278:13417-13421.

26. Morgans CW, Kensel-Hammes P, Hurley JB et al. Loss of the Synaptic Vesicle Protein SV2B results in reduced neurotransmission and altered synaptic vesicle protein expression in the retina. PLoS One 2009;4:e5230. 
27. Huber TB, Hartleben B, Kim J et al. Nephrin and CD2AP associate with phosphoinositide 3-OH kinase and stimulate AKT-dependent signaling. Mol Cell Biol 2003;23:4917-4928.

28. Zhu J, Sun N, Aoudjit $L$ et al. Nephrin mediates actin reorganization via phosphoinositide 3-kinase in podocytes. Kidney Int 2008;73: 556-566.

29. Wang H, Misaki T, Taupin V et al. GIV/Girdin Links Vascular Endothelial Growth Factor Signaling to Akt Survival Signaling in Podocytes Independent of Nephrin. J Am Soc Nephrol 2014;26:314-327.

30. Armelloni $\mathrm{S}$, Calvaresi $\mathrm{N}$, Ikehata $\mathrm{M}$ et al. Proteinuria and glomerular damage in Rab3A knockout mice chronically fed a high-glucose diet. Nephron Exp Nephrol 2012;120:e69-e80.

31. Lazzell DR, Belizaire R, Thakur $P$ et al. SV2B regulates synaptotagmin 1 by direct interaction. J Biol Chem 2004;279:52124-52131.

32. Yao J, Nowack A, Kensel-Hammes $P$ et al. Cotrafficking of SV2 and synaptotagmin at the synapse. J Neurosci 2010;30:5569-5578.
33. Tian $\mathrm{D}$, Jacobo SM, Billing $\mathrm{D}$ et al. Antagonistic regulation of actin dynamics and cell motility by TRPC5 and TRPC6 channels. Sci Signal 2010;3:ra77.

34. Schaldecker T, Kim S, Tarabanis $C$ et al. Inhibition of the TRPC5 ion channel protects the kidney filter. J Clin Invest 2013;123:5298-5309.

35. Burford JL, Villanueva K, Lam L et al. Intravital imaging of podocyte calcium in glomerular injury and disease. J Clin Invest 2014;124: 2050-2058.

36. Perin MS. The $\mathrm{COOH}$ terminus of synaptotagmin mediates interaction with the neurexins. J Biol Chem 1994;269:8576-8581.

37. Fukuda M, Moreira JE, Liu V et al. Role of the conserved WHXL motif in the $C$ terminus of synaptotagmin in synaptic vesicle docking. Proc Natl Acad Sci USA 2000;97:14715-14719.

38. Giardino L, Armelloni S, Corbelli A et al. Podocyte glutamatergic signaling contributes to the function of the glomerular filtration barrier. J Am Soc Nephrol 2009;20:1929-1940. 